\title{
Uninorms Having An Identity and An Annihilator on Bounded Lattices
}

\author{
Sinırlı Kafesler Üzerinde Bir Birime ve Bir Sifirlayana Sahip Uninormlar
}

\section{Gül Deniz ÇAYLI*}

Department of Mathematics, Faculty of Science, Karadeniz Technical University, 61080, Trabzon

\author{
• Geliş tarihi / Received: 23.06.2018 • • Düzeltilerek geliş tarihi / Received in revised form: 06.11.2018 • Kabul tarihi / Accepted: 19.11 .2018
}

\begin{abstract}
Uninorms defined on bounded lattices are an important generalization of triangular norms and triangular conorms and these operators allow the identity to be any point in a bounded lattice. In this study, uninorms on bounded lattices are studied. It is proposed a method to characterize uninorms on bounded lattices having an identity and an annihilator on bounded lattices and some basic properties of such uninorms are investigated. Moreover, an example is provided to illustrate the feasibility of the proposed method.
\end{abstract}

Keywords: Identity, Annihilator, Bounded lattice, Uninorm

$\ddot{O} z$

Sinırl kafesler üzerinde tanımlanan uninormlar, üçgensel normların ve üçgensel konormların önemli bir genelleştirmesidir ve bu operatörler, birimin sınırlı kafesin herhangi bir noktasında olmasına olanak să̆larlar. Bu çalışmada, sınırlı kafesler üzerinde uninormlar konusu üzerine çalışlmıştır. Sinırlı kafesler üzerinde bir birime ve sıfirlayana sahip uninormları karakterize etmek için bir metot önerildi ve bu şekildeki uninormların bazı temel özellikleri araştırıldı. Ayrıca, önerilen metotun uygulanabilirliğini göstermek için bir örnek verildi.

Anahtar kelimeler: Birim, Sifirlayan, Sinırlı Kafes, Uninorm

* Gül Deniz ÇAYLI; guldeniz.cayli@ktu.edu.tr; Tel: (0462) 37725 66; orcid.org/0000-0002-7918-9752 


\section{Introduction}

The concept of uninorm appeared in (Yager and Rybalov, 1996) and comprehensively discussed in (Fodor et al., 1997) as a particular sort of aggregation operators which is a generalization of the concepts of triangular norm and triangular conorm. Uninorms on real unit interval with the identity which is any point in $[0,1]$ such that uninorms having an identity 0 are known as triangular conorms and uninorms having an identity 1 are known as triangular norms. Uninorms are attractive due to their structure as a particular union of triangular norms and triangular conorms that was demonstrated beneficial in numerous areas like aggregation, expert systems, multicriteria decision support, neural networks, fuzzy logic and fuzzy system modeling (Tsadiras and Margaritis, 1998; De Baets and Fodor, 1999; Yager, 2001; Yager, 2003; Yager and Kreinovich, 2003; Beliakov et al., 2007; Gabbay and Metcalfe, 2007). Along with this several application areas, these operators were also discussed from the merely theoretical aspect. By this means, one of the aspect is the characterizations of uninorms having an identity (Drewniak and Drygaś, 2002; Grabisch et al., 2009; Mesiarová-Zemanková, 2015; Drygaś et al., 2017).

In (Karaçal and Mesiar, 2015), two methods in order to show that a uninorm via the presence of triangular norms and triangular conorms in a bounded lattice always exist were proposed. Via the proposed methods, the least uninorm and the greatest uninorm having an identity was obtained. In (Çaylı et al., 2016), several additional methods to characterize uninorms having an identity in a bounded lattice were introduced. Furthermore, a uninorm on some bounded lattices which an identity $\mathrm{g}$ is incomparable with an annihilator $k$ need not always exist was proved. The uninorms on bounded lattices were also discussed by several researchers in other studies (Bodjanova and Kalina, 2014; Çaylı and Karaçal, 2017; Aşıc1, 2018; Çaylı and Drygaś, 2018).

In this paper, uninorms on bounded lattices are discussed. By taking a bounded lattice $L$, the possible values of uninorms having an annihilator $k$ and an identity $e$ from $L$ are investigated as well as an examination of their basic characteristics are provided. Based on these characteristics, it is showed that there need not always be existence a uninorm on $L$ having an identity $e$ and an annihilator $k$ once $k$ and $e$ are comparable with each other (it was proposed the case of $k$ and $e$ are incomparable with each other in (Çaylı et al.,
2016). Also, a method for constructing uninorms having an identity $e$ and an annihilator $k$ on $L$ such that $k$ is incomparable with $e$ is introduced under an additional assumption that all elements in $L$ different from $e$ are comparable with $k$.

This paper comprises of three parts. After some basic results concerning bounded lattices and uninorms on them, in Section 3, some properties of monotone operations on a bounded lattice $L$ are examined for an annihilator $k$ and an identity $e$, where $e, k \neq 0,1$. The presence of uninorms on $L$ which an identity $e$ is incomparable with an annihilator $k$ is investigated. Moreover, a construction method yielding uninorms on bounded lattices having an annihilator and an identity such that these elements are incomparable each other is proposed under an additional constraint. Moreover, an illustrative example is added to clearly understand our method. Finally, some conclusions are given.

\section{Preliminaries}

In this part, some main results dealing with bounded lattices and uninorms, triangular norms, triangular conorms defined on them are given.

A lattice $(L, \leq)$ is bounded once $L$ has the top element and bottom element represented, respectively, 1 and 0 , namely, there are two elements 1,0 , where $0 \leq a \leq 1$ for all $a \in L$.

Throughout this paper, $L$ always represents any given general bounded lattice with the top element 1 and bottom element 0 unless otherwise stated.

Definition 2.1. (Birkhoff, 1967) Let $p, q \in L$. If $p$ and $q$ are incomparable, this is denoted by $p \| q$. The set of elements the fact that are incomparable with $k \in L$ is denoted by $I_{k}$. So, $I_{k}=\{p \in L \mid p \|$ $k\}$. In the similar way, the set of elements the fact that are incomparable with $e \in L$ is denoted by $I_{e}$. So, $I_{e}=\{p \in L \mid p \| e\}$.

Definition 2.2. (Birkhoff, 1967) Let $p, q \in L$ and $p \leq q$. In that case, it is defined a subinterval $[p, q]$ in $L$ as below:

$[p, q]=\{a \in L: p \leq a \leq q\}$.

In the same way, it is defined $] p, q]=\{a \in L$ : $p<a \leq q\}, \quad[p, q[=\{a \in L: p \leq a<q\} \quad$ and ]$p, q[=\{a \in L: p<a<q\}$.

Definition 2.3. (Çayli et al., 2016) Define a function $F: L^{2} \rightarrow L$. 
i) A fixed element $e \in L$ is called an identity (sometimes called neutral element) of $F$ provided that $F(p, e)=F(e, p)=p$ for all $p \in L$.

ii) A fixed element $k \in L$ is called an annihilator (sometimes called absorbing element or zero element) of $F$ provided that $F(p, k)=F(k, p)=$ $k$ for all $p \in L$.

Definition 2.4. (Karacal and Mesiar, 2015; Cayl1 et al., 2016) If an operation $U$ on $L$ having an identity is associative, commutative, monotone, then it is called a uninorm on $L$ (briefly uninorm, where $L$ is fixed).

Denote the set of all uninorms having an identity $e$ in $L$ by $\mathcal{P}(e)$.

Definition 2.5. (Karacal and Mesiar, 2015; Çayl1 et al., 2016) If an operation $T$ on L having an identity 1 is associative, commutative, monotone, then it is called a triangular norm (t-norm, briefly) on $L$.

Definition 2.6. (Așıc1 and Karaçal, 2016; Aşıc1, 2017; Cayl1, 2018a,b) If an operation $S$ on $L$ having an identity 0 is associative, commutative, monotone, then it is called a triangular conorm ( $t$ conorm, briefly) on $L$.

Example 2.7. The least triangular norm $T_{W}$ and the greatest triangular norm $T_{\wedge}$ on $L$ are defined as, respectively:

$T_{W}(p, q)= \begin{cases}q & \text { if } p=1 \\ p & \text { if } q=1 \\ 0 & \text { otherwise }\end{cases}$

$T_{\wedge}(p, q)=\inf \{p, q\}$

The least triangular conorm $S_{\mathrm{V}}$ and the greatest triangular conorm $S_{W}$ on $L$ are defined as, respectively:

$S_{\mathrm{V}}(p, q)=\sup \{p, q\}$

$S_{W}(p, q)= \begin{cases}q & \text { if } p=0 \\ p & \text { if } q=0 \\ 1 & \text { otherwise }\end{cases}$

The undermentioned sets is represented by $D_{k}$ and $D_{e}$, respectively: for $k, e \in L \backslash\{0,1\}$

$D_{k}=[0, k] \times[k, 1] \cup[k, 1] \times[0, k]$

and
$D_{e}=[0, e] \times[e, 1] \cup[e, 1] \times[0, e]$.

Denote the set of all uninorms on $L$ by $\mathcal{P}$ and define the order on $\mathcal{P}$ in the undermentioned: For the uninorms $U_{1}, U_{2} \in \mathcal{P}$

$U_{1} \leq U_{2} \Leftrightarrow U_{1}(a, b) \leq U_{2}(a, b)$ for all $a, b \in$ L.

It is obvious that $\mathcal{P}$ is a partially ordered set which has the top element and bottom element, respectively, $S_{W}$ and $T_{W}$.

In the same way, it can be clearly seen that each $\mathcal{P}(e)$ is a partially ordered set, too.

Proposition 2.8. (Karacal and Mesiar, 2015) Given a uninorm $U: L^{2} \rightarrow L$ for an identity $e \in L$ such that $e \neq 0,1$. In that case, it is obtained that

i) The restriction of $U$ on $[0, e]$ is a triangular norm.

ii) The restriction of $U$ on $[e, 1]$ is a triangular conorm.

Proposition 2.9. (Karacal and Mesiar, 2015) Given a uninorm $U: L^{2} \rightarrow L$ for an identity $e \in L$ such that $e \neq 0,1$. In that case, it is obtained the undermentioned properties:

i) $\inf \{p, q\} \leq U(p, q) \leq \sup \{p, q\}$ for all $(p, q) \in D_{e}$.

ii) $U(p, q) \leq p$ for all $p \in L$ and $q \in[0, e]$.

iii) $U(p, q) \leq q$ for all $p \in[0, e]$ and $q \in L$.

iv) $p \leq U(p, q)$ for all $p \in L$ and $q \in[e, 1]$.

v) $q \leq U(p, q)$ for all $p \in[e, 1]$ and $q \in L$.

\section{Uninorms having an annihilator $k$ and an identity $e$}

In this part, taking a bounded lattice $L$, some properties of monotone operations on $L$ having an annihilator $k$ and an identity $e$ in $L$ such that $e, k \neq 0,1$ are researched. Furthermore, a construction method for uninorms on $L$ having an annihilator $k$ and an identity $e$ in $L$ is proposed where $e \neq 0,1, \quad k \| e$ and all elements in $L$ different from $e$ are comparable with $k$.

The next Proposition 3.1 and Proposition 3.2 can be found in (Drygaś, 2004; Karaçal and Mesiar, 2015). 
Proposition 3.1. Consider the elements $e, k \in L$ such that $e, k \neq 0,1$ and $k \leq e$. If $\mathrm{F}$ is a monotone operation on $L$ having an annihilator $k$ and an identity $e$, in that case, it is obtained the undermentioned properties:

i) $k \leq F(p, q) \leq \inf \{p, q\}$ for all $(p, q) \in[k, e]^{2}$.

ii) $p \leq F(p, q) \leq q$ for all $(p, q) \in[k, e] \times[e, 1]$.

iii) $q \leq F(p, q) \leq p$ for all $(p, q) \in[e, 1] \times$ $[k, e]$.

iv) $k \leq F(p, q) \leq q$ for all $(p, q) \in[k, e] \times L$.

v) $k \leq F(p, q) \leq p$ for all $(p, q) \in L \times[k, e]$.

vi) $q \leq F(p, q)$ for all $(p, q) \in[e, 1] \times L$.

vii) $p \leq F(p, q)$ for all $(p, q) \in L \times[e, 1]$.

Proposition 3.2. Consider the elements $e, k \in L$ such that $e, k \neq 0,1$ and $e \leq k$. If $F$ is a monotone operation on $L$ having an annihilator $k$ and an identity $e$, in that case it is obtained the undermentioned properties:

i) $p \leq F(p, q) \leq q$ for all $(p, q) \in[0, e] \times[e, k]$.

ii) $q \leq F(p, q) \leq p$ for all $(p, q) \in[e, k] \times[0, e]$.

iii) $\sup \{p, q\} \leq F(p, q) \leq k$ for all $(p, q) \in$ $[e, k]^{2}$.

iv) $F(p, q) \leq q$ for all $(p, q) \in[0, e] \times L$.

v) $F(p, q) \leq p$ for all $(p, q) \in L \times[0, e]$.

vi) $q \leq F(p, q) \leq k$ for all $(p, q) \in[e, k] \times L$.

vii) $p \leq F(p, q) \leq k$ for all $(p, q) \in L \times[e, k]$.

Proposition 3.3. Given the elements $e, k \in L$ such that $e, k \neq 0,1$ and $k \leq e$. If there is an element $m$ in $L$ which $m \in[0, k[$, in that case there is no monotone operation on $L$ having an annihilator $k$ and an identity $e$.

Proof. Given an element $m$ in $L$ such that $m \in[0, k[$. Suppose that there is a monotone operation $F$ on $L$ having an annihilator $k$ and an identity $e$. Since $k \leq e$ and the fact that $k$ is an annihilator, it is obtained $k=F(m, k) \leq$ $F(m, e) \leq F(k, e)=k$. So, $F(m, e)=k$. Due to the fact that $e$ is an identity, then $F(m, e)=m$. Therefore, it is obtained $k=m$. This is a contradiction. Therefore, there is no monotone operation $F$ on $L$ having an annihilator $k$ and an identity $e$ once there is an element $m$ in $L$ which $m \in[0, k[$.

Proposition 3.4. Given the elements $e, k \in L$ such that $e, k \neq 0,1$ and $e \leq k$. If there is an element $m$ in $L$ which $m \in] k, 1]$, in that case there is no monotone operation on $L$ having an annihilator $k$ and an identity $e$.

It is clearly proved in the same way to Proposition 3.3.

Remark 3.5. Consider the elements $e, k \in L$ such that $e, k \neq 0,1$.

i) If $k \leq e$ and there is an element $l$ in $L$ which $l \in[0, k[$, there does not exist any uninorm having an annihilator $k$ and an identity $e$ in $L$ due to Proposition 3.3.

ii) If $e \leq k$ and there is an element $l$ in $L$ which $l \in] k, 1]$, there does not exist any uninorm having an annihilator $k$ and an identity $e$ in $L$ due to Proposition 3.4..

iii) If $e \in I_{k}$, it can be seen that there does not exist any uninorm on $L$ which contains the sublattices that are isomorphic to the sublattices depicted in (Çaylı et al., 2016) having an annihilator $k$ and an identity $e$ in $L$.

Let us introduce these sublattices that is given in Theorem 3.6 and Theorem 3.7 proposed by (Çayl1 et al., 2016).

Theorem 3.6. (Çayl1 et al., 2016) If $L$ comprises a sublattice which is isomorphic to the sublattice depicted with the given order in Figure 1, in the present case, there does not exist any uninorm having an annihilator $k$ and an identity $e$ in $L$.

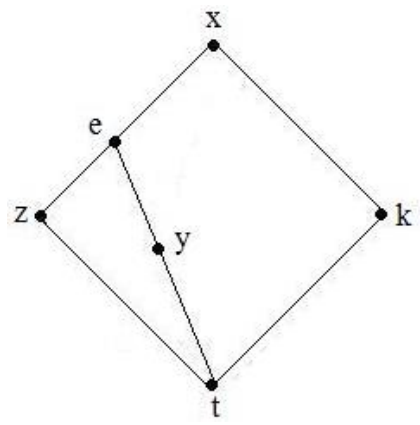

Figure 1. The lattice $L$ 
Theorem 3.7. (Çayll et al., 2016) If $L$ comprises a sublattice which is isomorphic to the sublattice depicted with the given order in Figure 2 in the present case, there does not exist any uninorm having an annihilator $k$ and an identity $e$ in $L$.

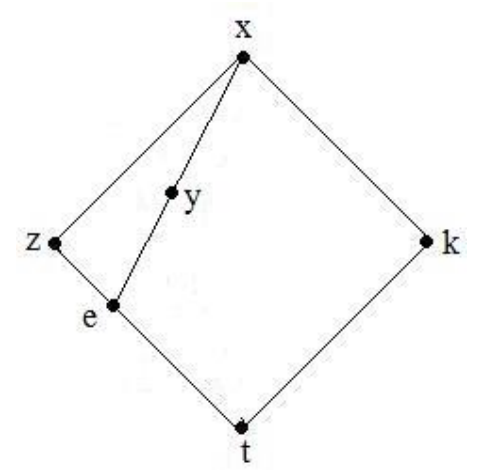

Figure 2. The lattice $L$

Remark 3.8. Consider the elements $e, k \in L$ such that $e, k \neq 0,1$ and $k \in I_{e}$. Note that there are some elements different from $e$ which are incomparable with the annihilator $k$ in bounded lattices depicted by Figure 1 and Figure 2. A natural question occurs: If all elements in $L$ different from $e$ is comparable with $k$, is there always a uninorm $U$ on $L$ having an annihilator $k$ and an identity $e$ ?

It can be seen that the below theorem provides a positive answer to the above question.

Theorem 3.9. Take the elements $e, k \in L f$ such that $e, k \neq 0,1$ and $k \in I_{e}$. If all elements in $L$ different from $e$ are comparable with $k$, in the present case, the undermentioned function $U_{l}: L^{2} \rightarrow L$

$U_{l}(p, q)= \begin{cases}\inf \{p, q\} & \text { if } p, q \in[0, k[ \\ \sup \{p, q\} & \text { if } p, q \in] k, 1] \\ k & \text { if } p, q \in D_{k} \\ p & \text { if } p \in L \text { and } q=e \\ q & \text { if } p=e \text { and } q \in L\end{cases}$

is a uninorm on $L$ having an annihilator $k$ and an identity $e$.

Proof. i) Monotonicity: It is demonstrated the fact that if $p \leq q$ then $U_{l}(p, t) \leq U_{l}(q, t)$ for all $t \in L$. If $t=e$, in that case it is obtained $U_{l}(p, t)=$ $U_{l}(p, e)=p \leq q=U_{l}(q, e)=U_{l}(q, t)$ for all $p, q \in L$.

If $t=k$, in that case it is obtained $U_{l}(p, t)=$ $U_{l}(p, k)=k=U_{l}(q, k)=U_{l}(q, t) \quad$ for $\quad$ all $p, q \in L$.
So, it is examined other all possible cases.

Case 1.. Let $p<k$. In the present case,

Case 1.1.. $q<k$,

Case 1.1.1.. $t<k$,

$U_{l}(p, t)=\inf \{p, t\} \leq \inf \{q, t\}=U_{l}(q, t)$

Case 1.1.2.. $t>k$,

$U_{l}(p, t)=k=U_{l}(q, t)$

Case 1.2.. $q>k$,

Case 1.2.1.. $t<k$

$U_{l}(p, t)=\inf \{p, t\} \leq k=U_{l}(q, t)$

Case 1.2.2.. $t>k$,

$U_{l}(p, t)=k \leq \sup \{q, t\}=U_{l}(q, t)$

Case 1.3.. $q=e$,

Case 1.3.1.. $t<k$

$U_{l}(p, t)=\inf \{p, t\} \leq t=U_{l}(q, t)$

Case 1.3.2.. $t>k$,

$U_{l}(p, t)=k \leq t=U_{l}(q, t)$

Case 1.4.. $q=k$

Case 1.4.1.. $t<k$,

$U_{l}(p, t)=\inf \{p, t\} \leq k=U_{l}(q, t)$

Case 1.4.2.. $t>k$,

$U_{l}(p, t)=k=U_{l}(q, t)$

Case 2.. Let $p>k$. In the present case, $q>k$.

Case 2.1.. $t<k$,

$U_{l}(p, t)=k=U_{l}(q, t)$

Case 2.2.. $t>k$,

$U_{l}(p, t)=\sup \{p, t\} \leq \sup \{q, t\}=U_{l}(q, t)$

Case 3.. Let $=e$. In the present case,

Case 3.1.. $q>k$,

Case 3.1.1.. $t<k$,

$U_{l}(p, t)=t \leq k=U_{l}(q, t)$

Case 3.1.2.. $t>k$,

$U_{l}(p, t)=t \leq \sup \{q, t\}=U_{l}(q, t)$

Case 3.2.. $q=e$, then for all $t \in L$, it is obtained $U_{l}(p, t)=U_{l}(e, t)=t=U_{l}(e, t)=U_{l}(q, t)$

Case $4 .$. Let $=k$. In the present case,

Case 4.1.. $q>k$,

Case 4.1.1.. $t<k$,

$U_{l}(p, t)=k=U_{l}(q, t)$

Case 4.1.2.. $t>k$,

$U_{l}(p, t)=k \leq \sup \{q, t\}=U_{l}(q, t)$

Case 4.2.. $q=k$, then it is obtained $t \in L$.

$U_{l}(p, t)=U_{l}(k, t)=k=U_{l}(k, t)=U_{l}(q, t)$

ii) Associativity: For all $p, q, t \in L$, it is proved $U_{l}\left(p, U_{l}(q, t)\right)=U_{l}\left(U_{l}(p, q), t\right)$.

If the at least one of the elements $p, q, t$ is equal to $k$, then it is obtained $U_{l}\left(p, U_{l}(q, t)\right)=$ $U_{l}\left(U_{l}(p, q), t\right)$.

If the at least one of the elements $p, q, t$ is equal to $e$, then it is obtained $U_{l}\left(p, U_{l}(q, t)\right)=$ $U_{l}\left(U_{l}(p, q), t\right)$. 
So, it is examined the case that not the elements $p, q, t$ is equal to $k$ and $e$.

Case 1.. Let $p<k$. In the present case,

Case 1.1.. $q<k$,

Case 1.1.1.. $t<k$,

$$
\begin{aligned}
U_{l}\left(p, U_{l}(q, t)\right) & =U_{l}(p, \inf \{q, t\})=\inf \{p, q, t\} \\
& =U_{l}(\inf \{p, q\}, t) \\
& =U_{l}\left(U_{l}(p, q), t\right)
\end{aligned}
$$

Case 1.1.2.. $t>k$,

$$
U_{l}\left(p, U_{l}(q, t)\right)=U_{l}(p, k)=k=U_{l}(\inf \{p, q\}, t)
$$

Case 1.2.. $\mathrm{q}>k$

$$
=U_{l}\left(U_{l}(p, q), t\right)
$$

Case 1.2.1.. $t<k$,

$$
\begin{aligned}
U_{l}\left(p, U_{l}(q, t)\right) & =U_{l}(p, k)=k=U_{l}(k, t) \\
& =U_{l}\left(U_{l}(p, q), t\right)
\end{aligned}
$$

Case 1.2.2.. $t>k$,

$$
\begin{aligned}
U_{l}\left(p, U_{l}(q, t)\right) & =U_{l}(p, \sup \{q, t\})=k=U_{l}(k, t) \\
& =U_{l}\left(U_{l}(p, q), t\right)
\end{aligned}
$$

Case 2.. Let $p>k$. In the present case,

Case 2.1.. $q<k$

Case 2.1.1.. $t<k$

$$
\begin{gathered}
U_{l}\left(p, U_{l}(q, t)\right)=U_{l}(p, \inf \{q, t\})=k=U_{l}(k, t) \\
=U_{l}\left(U_{l}(p, q), t\right)
\end{gathered}
$$

Case 2.1.2.. $t>k$,

$$
\begin{gathered}
U_{l}\left(p, U_{l}(q, t)\right)=U_{l}(p, k)=k=U_{l}(k, t) \\
=U_{l}\left(U_{l}(p, q), t\right)
\end{gathered}
$$

Case 2.2.. $q>k$,

Case 2.2.1.. $t<k$,

$$
\begin{gathered}
U_{l}\left(p, U_{l}(q, t)\right)=U_{l}(p, k)=k=U_{l}(\sup \{p, q\}, t) \\
=U_{l}\left(U_{l}(p, q), t\right)
\end{gathered}
$$

Case 2.2.2.. $t>k$,

$$
\begin{aligned}
U_{l}\left(p, U_{l}(q, t)\right) & =U_{l}(p, \sup \{q, t\})=\sup \{p, q, t\} \\
& =U_{l}(\sup \{p, q\}, t) \\
& =U_{l}\left(U_{l}(p, q), t\right)
\end{aligned}
$$

It is clear to prove that the commutativity holds, $k$ is an annihilator and $e$ is a an identity of $U_{l}$. So, $U_{l}$ is a uninorm having an annihilator $k$ and an identity $e$ on $L$.

Corollary 3.10. Take the elements $e, k \in L$ such that $e, k \neq 0,1$ and $k \in I_{e}$. Let all elements in $L$ different from $e$ be comparable with $k$.

i) If $k$ is a coatom of $L$, from Theorem 3.9 it is obtained the uninorm $U_{l}$ is as below

$$
\begin{aligned}
& U_{l}(p, q) \\
& = \begin{cases}\inf \{p, q\} & \text { if } p, q \in[0, k[ \\
1 & \text { if } p, q \in] k, 1] \\
k & \text { if } p, q \in[0, k] \times\{k, 1\} \cup\{k, 1\} \times[0, k] \\
p & \text { if } p \in L \text { and } q=e \\
q & \text { if } p=e \text { and } q \in L\end{cases}
\end{aligned}
$$

ii) If $k$ is a atom of $L$, from Theorem 3.9 it is obtained the uninorm $U_{l}$ is as below

$$
\begin{aligned}
& U_{l}(p, q) \\
& = \begin{cases}0 & \text { if } p, q \in[0, k[ \\
\sup \{p, q\} & \text { if } p, q \in] k, 1] \\
k & \text { if } p, q \in[k, 1] \times\{0, k\} \cup\{0, k\} \times[k, 1] \\
p & \text { if } p \in L \text { and } q=e \\
q & \text { if } p=e \text { and } q \in L\end{cases}
\end{aligned}
$$

Example 3.11. Take a lattice $L=\{0, p, q, r, k, e, m, t, 1\}$ with Hasse diagram shown in Figure 3 and consider a function $U: L^{2} \rightarrow L$ as Table 1 . Then $U$ is a uninorm having an annihilator $k$ and an identity $e$ in $L$ via Theorem 3.9.

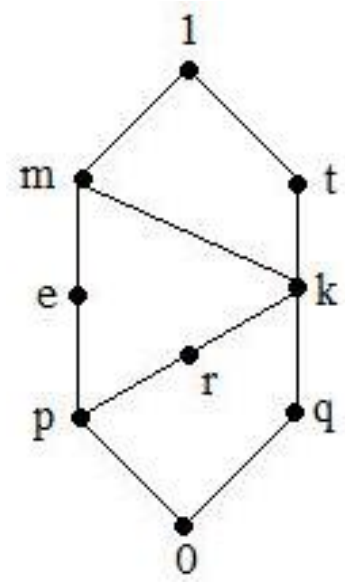

Figure 3. The lattice $L$

Table 2. The uninorm $U$ on $L$

\begin{tabular}{|c|c|c|c|c|c|c|c|c|c|}
\hline$U$ & 0 & $p$ & $q$ & $r$ & $e$ & $k$ & $m$ & $t$ & 1 \\
\hline 0 & 0 & 0 & 0 & 0 & 0 & $k$ & $k$ & $k$ & $k$ \\
\hline$p$ & 0 & $p$ & 0 & $p$ & $p$ & $k$ & $k$ & $k$ & $k$ \\
\hline $\mathrm{q}$ & 0 & 0 & $q$ & 0 & $q$ & $k$ & $k$ & $k$ & $k$ \\
\hline$r$ & 0 & $p$ & 0 & $r$ & $r$ & $k$ & $k$ & $k$ & $k$ \\
\hline$e$ & 0 & $p$ & $q$ & $r$ & $e$ & $k$ & $m$ & $t$ & 1 \\
\hline$k$ & $k$ & $k$ & $k$ & $k$ & $k$ & $k$ & $k$ & $k$ & $k$ \\
\hline$m$ & $k$ & $k$ & $k$ & $k$ & $m$ & $k$ & $m$ & 1 & 1 \\
\hline$t$ & $k$ & $k$ & $k$ & $k$ & $t$ & $k$ & 1 & $t$ & 1 \\
\hline 1 & $k$ & $k$ & $k$ & $k$ & 1 & $k$ & 1 & 1 & 1 \\
\hline
\end{tabular}

Remark 3.12. Consider the elements $e, k \in L$ which $e, k \neq 0,1$ and $k \in I_{e}$. It is known that there need not always exist a uninorm on $L$ having an annihilator $k$ and an identity $e$ due to the fact that Theorem 3.6 and Theorem 3.7, if it is choosen the special elements in $L$ that are different from $e$ and incomparable with $k$. For this reason, it is introduced in Theorem 3.9 a method for constructing uninorms on $L$ having an annihilator $k$ and an identity $e$, under a constraint that all 
elements in $L$ different from $e$ are comparable with $k$. In the present case, another elemental question occurs: once there exist some elements in $L$ different from $e$ that are incomparable with $k$, is there a uninorm having an annihilator $k$ and an identity $e$ on $L$. It is provided a positive example approving to the above hypothesis.

Let us first research the possible values of $\mathcal{P}(e)$ having the indicated an annihilator $k$ which $e \in I_{k}$ for some special conditions.

Proposition 3.13. Take the elements $e, k \in L$ which $e, k \neq 0,1$ and $k \in I_{e}$, a uninorm $U$ having an annihilator $k$ and an identity $e$ on $L$ and $l, d \in L \quad$ which $l \in[0, e[, \quad l \in[0, k[, d \in] e, 1]$, $d \in I_{k}$ and $\inf \{d, k\}=l$. In this case, it is obtained $U(l, d)=l$ and $U(d, d)<k$ or $U(d, d) \in I_{k}$.

Proof. Due to the monotonicity of $U$ and that $e$ is an identity, it is obtained $l=U(l, e) \leq U(l, d) \leq$ $U(e, d)=d$. Moreover, since $k$ is an annihilator, it holds $U(l, d) \leq U(k, d)=k$. So, it is obtained $l \leq U(l, d) \leq \inf \{d, k\}=l$, i.e. $U(l, d)=l$. Due to the associativity of $U$, it is obtained $l=$ $U(l, d)=U(U(l, d), d)=U(l, U(d, d))$. Suppose that $U(d, d) \geq k$. Then it is obtained a contradiction that $l \geq k$. So, it is $U(d, d)<k$ or $U(d, d) \in I_{k}$.

Proposition 3.14. Take the elements $e, k \in L$ which $e, k \neq 0,1$ and $k \in I_{e}$, a uninorm $U$ having an annihilator $k$ and an identity $e$ on $L$ and $l, d, r \in L \quad$ which $\quad l \in[0, e[, l \in[0, k[, d \in] e, 1]$, $d \in I_{k}, \inf \{d, k\}=l, r>e, r \in I_{k}$ and $\inf \{r, k\}=$ $l$. In the present case, it is obtained $U(d, r)<k$ or $U(d, r) \in I_{k}$.

Proof. From Proposition 3.14, it is $l=U(l, d)$ and $l=U(l, r)$. By the associativity of $U$, it is obtained $\quad l=U(l, r)=U(U(l, d), r)=$ $U(l, U(d, r))$. Suppose that $U(d, r) \geq k$. Then it is obtained a contradiction that $l \geq k$. So, it is $U(d, r)<k$ or $U(d, r) \in I_{k}$.

Proposition 3.15. Take the elements $e, k \in L$ which $e, k \neq 0,1$ and $k \in I_{e}$, a uninorm $U$ having an annihilator $k$ and an identity $e$ on $L$ and $l, d, r \in L$ which $r \in[0, e[, r \in[0, k[, l \in] e, 1]$, $l \in I_{k}, \inf \{l, k\}=r$ and $d<k$. In this case it is obtained $U(l, d)<k$ or $U(l, d) \in I_{k}$.

Proof. From Proposition 3.14, the monotonicity of $U$ and that $e$ is an identity, it is obtained $U(U(r, l), d)=U(r, d) \leq U(e, d)=d$. Suppose that $U(l, d) \geq k$. Then it holds $U(r, U(l, d)) \geq k$. By using the associativity of $U$, it is obtained $k \leq d$. This is a contradiction. Therefore, it is obtained either $U(l, d)<k$ or $U(l, d) \in I_{k}$.

Proposition 3.16. Take the elements $e, k \in L$ that $e, k \neq 0,1$ and $k \in I_{e}$, a uninorm $U$ having an annihilator $k$ and an identity $e$ on $L$. In the present case, it is obtained $U(0, r) \leq \inf \{r, k\}$ and $U(1, r) \geq \sup \{r, k\}$ for all $r \in L$.

Proof. Due to the monotonicity of $U$ and $e$ is an identity and $k$ is an annihilator, it is obtained $U(0, r) \leq U(e, r)=r$ and $U(0, r) \leq U(k, r)=$ $k$. So, $U(0, r) \leq \inf \{r, k\}$. Similarly, it is obtain that $U(1, r) \geq \sup \{r, k\}$.

Proposition 3.17. Take the elements $e, k \in L$ which $e, k \neq 0,1$ and $k \in I_{e}$, a uninorm $U$ having an annihilator $k$ and an identity $e$ on $L$ and $l, d \in L$ which $l \in\left[0, e\left[, d \in I_{e}, \sup \{l, k\}=1\right.\right.$ and $\sup \{d, k\}=1$. In this case, it is obtained $U(l, d) \neq 0$.

Proof. Suppose that $U(l, d)=0$. By Proposition 3.16 , it is obtained $U(1, l)=1$ and $U(1, d)=1$. Then it is had $U(U(1, d), l)=U(1, l)=1$. Moreover, it is obtained $U(1, U(l, d))=$ $U(1,0)=k$ by using the commutativity of $U$. This is a contradiction with the assocaitivity of $U$. So, $U(l, d) \neq 0$.

Proposition 3.18. Take the elements $e, k \in L$ which $e, k \neq 0,1$ and $k \in I_{e}$, a uninorm $U$ having an annihilator $k$ and an identity $e$ on $L$ and $l, d \in L$ which $l \in] e, 1], d \in I_{e}, \inf \{l, k\}=0$ and $\inf \{d, k\}=0$. In this case, it is obtained $U(l, d) \neq$ 1.

It can be demonstrated as dual of Proposition 3.17 .

By using Proposition 3.14-3.17 and considering a bounded lattice $L$ with Hasse diagram shown in Figure 4 in the undermentioned example, it can be defined a uninorm $U$ on $L$ for an annihilator $k$ and an identity $e$, although there exist the elements in $L$ which are different from $e$ and incomparable with $k$.

Example 3.19. Take a lattice $L=\{0, x, t, y, k, e, 1\}$ with Hasse diagram shown in Figure 4 and consider a function $U: L^{2} \rightarrow L$ as Table 2. However, there are some elements $y, t, x \in L$ that are incomparable with $k, U$ is a 
uninorm with an annihilator $k$ and an identity $e$ on $L$.

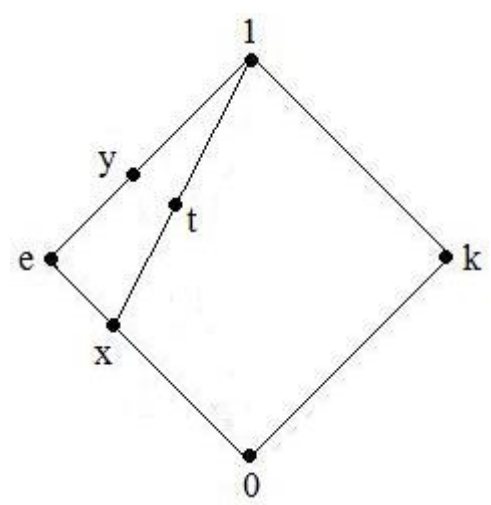

Figure 4. The lattice $L$

Table 2. The uninorm $U$ on $L$

\begin{tabular}{|c|c|c|c|c|c|c|c|}
\hline$U$ & 0 & $x$ & $e$ & $t$ & $k$ & $y$ & 1 \\
\hline 0 & 0 & 0 & 0 & 0 & $k$ & 0 & $k$ \\
\hline$x$ & 0 & $x$ & $x$ & $t$ & $k$ & $x$ & 1 \\
\hline$e$ & 0 & $x$ & $e$ & $t$ & $k$ & $y$ & 1 \\
\hline$t$ & 0 & $t$ & $t$ & $t$ & $k$ & $t$ & 1 \\
\hline$k$ & $k$ & $k$ & $k$ & $k$ & $k$ & $k$ & $k$ \\
\hline$y$ & 0 & $x$ & $y$ & $t$ & $k$ & $y$ & 1 \\
\hline 1 & $k$ & 1 & 1 & 1 & $k$ & 1 & 1 \\
\hline
\end{tabular}

\section{Concluding remarks}

In this study, the structure of uninorms on bounded lattices having an annihilator and an identity is discussed. It has been also investigated some properties of monotone operations on $L$ that have an annihilator and an identity. As byproduct, it has been shown that it needs not exist a uninorm having an annihilator $k$ and an identity $e$ on $L$ once $k \leq e$ or $e \leq k$. In (Çaylı et al., 2016) considering any bounded lattice $L$, it was presented the results that there need not always exist a uninorm $U \in \mathcal{P}(e)$ for an annihilator $k$ once $k \in I_{e}$ in Theorem 3.6 and Theorem 3.7. Note that it has been showed there need not be a uninorm $U \in \mathcal{P}(e)$ having an annihilator in Theorem 3.6 and Theorem 3.7 in the case of that it is chosen the special elements in $L$ are incomparable with an annihilator $k$. For this reason, it has been introduced a method to characterize uninorms having an annihilator $k$ and an identity $e$ on $L$, where $e, k \neq 0,1, k \in I_{e}$ and all elements in $L$ different from $e$ is comparable with $k$. Moreover, it has been exemplified that a uninorm having an annihilator $k$ and an identity $e$ on a bounded lattice $L$ can exist while there exist the elements in $L$ different from $e$ are incomparable with $k$. As a future study, one can consider whether it is possible to characterize a uninorm $U \in \mathcal{P}(e)$ with an annihilator $k$ under which additional constraints on $L$ and/or the elements in $L$ different from $e$ once there exist the elements in $L$ different from $e$ which is incomparable with $k$.

\section{References}

Aşıc1, E. and Karaçal, F., 2016. Incomparability with respect to the triangular order. Kybernetika, 52, $15-27$.

Aş1c1, E., 2017. An order induced by nullnorms and its properties. Fuzzy Sets and Systems, 325, 35-46.

Aş1c1, E., 2018. Some remarks on an order induced by uninorms, in: Kacprzyk, J., Szmidt, E., Zadrozny, S., Atanassov, K.T. and Krawczak, M. (Eds), Advances in Fuzzy Logic and Technology, Advances in Intelligent Systems and Computing, Springer, Cham, 641, 69-77.

Beliakov, G., Pradera, A. and Calvo, T., 2007. Aggregation Functions: A Guide for Practitioners: Berlin, Springer, 374 p.

Birkhoff, G., 1967. Lattice Theory: Providence, RI, American Mathematical Society Colloquium Publishing, $418 \mathrm{p}$.

Bodjanova, S. and Kalina, M., 2014. Construction of uninorms on bounded lattices. IEEE $12^{\text {th }}$ International Symposium on Intelligent Systems and Informatics, SISY 2014, 11-13 September 2014, Subotica, Serbia, pp. 61-66.

Çaylı, G.D. and Drygaś, P., 2018. Some properties of idempotent uninorms on a special class of bounded lattices. Information Sciences, 422, 352-363.

Çaylı, G.D. and Karaçal, F., 2017. Construction of uninorms on bounded lattices. Kybernetika, 53, 394-417.

Çaylı, G.D., 2018a. On a new class of t-norms and tconorms on bounded lattices. Fuzzy Sets and Systems, 332, 129-143.

Çayl1, G.D., 2018b. Uninorms that are neither conjunctive nor disjunctive on bounded lattices, in: Medina, J., et al. (Eds), Information Processing and Management of Uncertainty in Knowledge-Based Systems, Communications in Computer and Information Science, Springer, Cham, 853, 310-318.

Çaylı, G.D., Karaçal, F. and Mesiar, R., 2016. On a new class of uninorms on bounded lattices. Information Sciences, 367-368, 221-231. 
De Baets, B. and Fodor, J., 1999. Van Melle's combining function in MYCIN is a representable uninorm: an alternative proof. Fuzzy Sets and Systems, 104, 133-136.

Drewniak, J. and Drygaś, P., 2002. On a class of uninorms. International Journal of Uncertainty, Fuzziness and Knowledge-Based Systems, 10, $5-10$

Drygaś, P., 2004. Isotonic operations with zero element in bounded lattices, in: Atanassov, K. (Eds), Soft Computing Foundations and Theoretical Aspect, EXIT Warszawa, p. 181-190.

Drygaś, P., Qin, F. and Rak, E., 2017. Left and right distributivity equations for semi-t-operators and uninorms. Fuzzy Sets and Systems, 325, 21-34.

Fodor, J., Yager, R.R. and Rybalov, A., 1997. Structure of uninorms. International Journal of Uncertainty, Fuzziness and Knowledge-Based Systems, 5, 411-427.

Gabbay, D. and Metcalfe, G., 2007. Fuzzy logics based on $[0,1)$-continuous uninorms. Archive for Mathematical Logic, 46, 425-449.

Grabisch, M., Marichal, J.L., Mesiar, R. and Pap, E., 2009. Aggregation Functions: Cambridge, Cambridge University Press, 482p.
Karaçal, F. and Mesiar, R., 2015. Uninorms on bounded lattices. Fuzzy Sets and Systems, 261, $33-43$.

Mesiarová-Zemanková, A., 2015. Multi-polar tconorms and uninorms. Information Sciences, 301, 227-240.

Tsadiras, A.K. and Margaritis, R.G., 1998. The MYCIN certainly factor handling function as uninorm operator and its use as a threshold function in artificial neurons. Fuzzy Sets and Systems, 93, 263-274.

Yager, R.R. and Kreinovich, V., 2003. Universal approximation theorem for uninorm-based fuzzy systems modeling. Fuzzy Sets and Systems, 140, 331-339.

Yager, R.R. and Rybalov, A., 1996. Uninorms aggregation operators. Fuzzy Sets and Systems, 80, 111-120.

Yager, R.R., 2001. Uninorms in fuzzy systems modeling. Fuzzy Sets and Systems, 122 (1), 167-175.

Yager, R.R., 2003. Defending against strategic manipulation in uninorm-based multi-agent decision making. Fuzzy Sets and Systems, 140, 331-339. 